disease compared to blacks and NHW women.(Todorova, Tejada, \& Castaneda-Sceppa, 2014)These are cardiovascular risk factors that warrant further study in Puerto Rican women living on the island, but data are lacking. Objective: The purpose of this study is to evaluate the gender disparities in presentation, management, and outcomes in Puerto Rican Hispanic hospitalized for heart failure METHODS/STUDY POPULATION: To this end, we will perform a secondary analysis of data from the PR CardiovascularSurveillance Study (PRCSS). We will extract personal data from 4,461 medical records of patients admitted with heart failure (ICD-9 Codes 428) at 21 hospitals in Puerto Rico, during theyears2007, 2009 and 2011. For statistical methods, we will implement chi-square and t-tests at a significance level of 0.05 . RESULTS/ANTICIPATED RESULTS: We expect to find that women will have: fewer interventions, less optimized heart failure medication, higher BNP, older age of diagnosis, but paradoxically better outcome than male counterparts of the same age. DISCUSSION/SIGNIFICANCE OF IMPACT: With this study, we would like to raise awareness about gender-specific health disparities Puerto Rican Hispanic women with heart failure experience while hospitalized.

\section{Harnessing Community Paramedicine for Transformative Fall Prevention Solutions}

Carmen Quatman ${ }^{1}$, Melinda Gabriel ${ }^{2}$, David Wisner ${ }^{3}$, Mark Weade ${ }^{3}$, Jennifer Garvin ${ }^{1}$, Elizabeth Sheridan, Jessica Wiseman, and Catherine Quatman-Yates ${ }^{1}$

${ }^{1}$ The Ohio State University; ${ }^{2}$ Westerville Fire Division; ${ }^{3}$ Upper Arlington Fire Division

OBJECTIVES/GOALS: Healthcare costs for falls are expected to reach nearly 55 billion dollars annually in the US by 2020 . Leveraging 911 calls as trigger events to activate fall prevention solutions could transform our ability to identify high-risk individuals and significantly improve fall prevention strategies globally. METHODS/STUDY POPULATION: An innovative pilot program entitled Community-centered Fall Intervention Team (Community FIT). Community FIT that leverages 911 calls, implementation science approaches, community partnerships, and collaboration among multiple healthcare disciplines including physical therapists, community paramedics, physicians, and social service coordinators was used to design and implement a community paramedicine fall intervention program. 911 call reports from February 2016 - August 2019 were analyzed using time series analyses to measure community level outcomes in fall-related calls and transports. RESULTS/ANTICIPATED RESULTS: 224 grab bars were installed free of charge to local residents (averaging approximately $\$ 125$ per home for modifications). Over an 18 -month period, time series analysis indicated an approximate demonstrated a consistent drop in the average fall-related 911 calls per month from 11.6 to 4.5 calls (a change of $61.21 \%$ ) and a decrease of $58 \%$ in the transport rates to the hospital for fall-related 911 calls. 911 referrals to the community paramedicine program have also increased by $83 \%$, demonstrating increased activation of fall prevention strategies with Community FIT. DISCUSSION/SIGNIFICANCE OF IMPACT: Collectively, these pilot study results provide preliminary support for individual and system level improvements in fall prevention by leveraging 911 calls to activate a community medicine fall prevention program. Future studies are needed to determine reach, long-term effectiveness, and sustainability of the program.
CONFLICT OF INTEREST DESCRIPTION: Johnson \& Johnson Hip Fracture Advisory Board (not related to project submission)

Implementation of an Opt-Out recruitment policy at Duke University School of Medicine

Michelle Mack ${ }^{1}$, Jamie Roberts ${ }^{1}$, Dalia Mack ${ }^{2}$, Steven Shipes ${ }^{3}$, Stephanie A. Freel ${ }^{1}$, Marissa Stroo ${ }^{2}$, Roy McDonald ${ }^{3}$, and Denise Snyder ${ }^{1}$

${ }^{1}$ Duke University; ${ }^{2}$ Duke Office of Clinical Research; ${ }^{3}$ Duke University School of Medicine

OBJECTIVES/GOALS: In March 2019, Duke updated recruitment guidelines and adopted an "Engagement" policy that requires patients to 'opt-out' of communications regarding potential research participation. This created an opportunity to evaluate recruitment for ongoing clinical studies pre and post implementation. METHODS/STUDY POPULATION: Implementation of the new policy required new training for study teams, modification to recruitment plans, and expansion of ongoing efforts to improve direct-topatient messaging through EPIC/MyChart tools. The impact of this new policy on overall recruitment was monitored and characterized both prior to and after implementation of the policy. Customized MyChart messages have been generated for over 22 studies, with a total of 41,386 messages sent to potential participants. RESULTS/ ANTICIPATED RESULTS: Only a small number of study teams have modified their recruitment plans with transition to the new policy. This may be related to lack of understanding about policy implementation, potential recruitment opportunities, required training, resource limitations, etc. However, our case study, TMIST, had an $48 \%$ improvement in average enrollment within the first 2 months of implementation, and an almost $40 \%$ improvement in recruitment efficiency. Since becoming an "opt-out" institution, 11 study teams have implemented direct-to-patient recruitment via the MyChart patient portal. One unintended consequence we've noted is several different study invitations to potential participants within some patient populations. DISCUSSION/SIGNIFICANCE OF IMPACT: The new policy allows study teams to engage in direct-to-patient outreach, leading to an increase in enrollment for some studies. Incorporation of direct-to-patient messaging strategies can be a cost and resource saving measure to improve recruitment. The need to recruit similar populations demonstrated that strategic, thoughtful approaches are needed.

4409

\section{Indiana Clinical \& Translational Science Monon} Collaborative - Community Impact Hubs

Sarah Wiehe ${ }^{1}$, David Craig ${ }^{2}$, Matthew Wilcox ${ }^{1}$, Emily Hardwick ${ }^{3}$, Carrie Lawrence ${ }^{4}$, Fiona Schicho ${ }^{3}$, and Brenda Hudson ${ }^{3}$ ${ }^{1}$ Indiana University School of Medicine; ${ }^{2}$ IUPUI; ${ }^{3}$ Indiana Clinical and Translational Sciences Institute; ${ }^{4}$ Indiana University

\section{OBJECTIVES/GOALS:}

- Conduct an environmental scan of Marion County (Indianapolis) neighborhoods using electronic medical record data, state health data, and social and economic data

- Develop strong network of community collaborators

- Conduct a thorough assessment for each targeted neighborhood by listening and understanding the pressing health issues 